\title{
VERTEX OPERATOR ALGEBRAS AND IRREDUCIBILITY OF CERTAIN MODULES FOR AFFINE LIE ALGEBRAS
}

\author{
DRAŽEN ADAMOVIĆ
}

\begin{abstract}
We find the connection between the representation theory of vertex operator algebra $L\left(k \Lambda_{0}\right)$ and the irreducibility of tensor products $\overline{V(\mu)} \otimes L\left(k \Lambda_{0}\right)$. In the case of affine Lie algebra $A_{1}^{(1)}$, on every admissible rational level we construct a family of irreducible modules having infinite-dimensional weight spaces.
\end{abstract}

\section{Introduction}

Let $\mathfrak{g}$ be a simple finite-dimensional Lie algebra and $\hat{\mathfrak{g}}$ the associated affine Lie algebra. Let $\overline{V(\mu)}$ be a loop module for $\hat{\mathfrak{g}}$ corresponding to irreducible finite-dimensional $\mathfrak{g}$-module $V(\mu)$ and let $L(\lambda)$ be an irreducible highest weight $\hat{\mathfrak{g}}-$ module. Then the tensor product $\overline{V(\mu)} \otimes L(\lambda)$ has infinite-dimensional weight spaces.

V. Chari and A. Pressley in $[\mathrm{CP}]$ studied these modules in the case when $L(\lambda)$ is the integrable highest weight module. They proved that the $\hat{\mathfrak{g}}$-module $\overline{V(\mu)} \otimes L(\lambda)$ is irreducible if the weight $\mu$ is "large" compared with $\lambda$. In this way their construction gave the first examples of irreducible $\hat{\mathfrak{g}}$-modules having infinite-dimensional weight spaces. The author in [A] studied the case of critical level and proved that at this level there is uncountably many irreducible modules of the type $\overline{V(\mu)} \otimes L(\lambda)$. In the proof of irreducibility theorem in [CP] some structural results for integrable modules were used. For irreducibility result in [A], author used the structure of center of the completed enveloping algebra at the critical level. From the results which we mentioned follows that for studying such modules at the arbitrary level $k$, we have to use some peculiarity of the representation theory of affine Lie algebra on this level. We will show that the language of vertex operator algebras can be used in the irreducibility analysis of these modules.

In this paper we will consider the tensor products $\overline{V(\mu)} \otimes L_{k}$, where $L_{k}=$ $L\left(k \Lambda_{0}\right)$ is the irreducible highest weight $\hat{\mathfrak{g}}-$ module with the highest weight $k \Lambda_{0}$ ( $L_{k}$ is sometimes called vacuum-module). On $\hat{\mathfrak{g}}-$ module $L_{k}$ we have the structure of simple vertex operator algebra (see [FZ], [DL], [MP]). The irreducible

Received February 11, 1997.

1991 Mathematics Subject Classification. 17B67.

Kew words and phrases. Affine Lie algebra, Vertex operator algebra, Zhu's algebra, Tensor products, Loop modules, Admissible representations. 
$L_{k}$-modules are in one-to-one correspondence with the irreducible modules for corresponding Zhu's algebra $A\left(L_{k}\right)$, introduced in [Z]. Since Zhu's algebra $A\left(L_{k}\right)$ can be identified as certain quotient of the universal enveloping algebra $U(\mathfrak{g})$ (see [FZ]), every $A\left(L_{k}\right)$-module is also $U(\mathfrak{g})$-module.

Our main theorem gives the irreducibility criterion for modules $\overline{V(\mu)} \otimes L_{k}$ in the terms of representations of algebra $A\left(L_{k}\right)$. We will prove the following theorem which shows that the question of irreducibility for modules $\overline{V(\mu)} \otimes L_{k}$ is closely related to the classification of finite-dimensional modules for Zhu's algebra $A\left(L_{k}\right)$.

Theorem 1. Let $k \in \mathbb{C}$. The $\hat{\mathfrak{g}}-\operatorname{module} \overline{V(\mu)} \otimes L_{k}$ is irreducible if and only if $V(\mu)$ is not a module for Zhu's algebra $A\left(L_{k}\right)$.

In the case of affine Lie algebra $A_{1}^{(1)}$ Theorem 1 and the classification of irreducible $L_{k}$-modules obtained in $[\mathrm{AM}]$ enable us to decide on irreducibility of $\overline{V(\mu)} \otimes L_{k}$ for every $k \in \mathbb{C}$. In particular, we have the following irreducibility result.

Theorem 2. Let $k=p / q \in \mathbb{Q}$ such that $q \in \mathbb{N}, p \in \mathbb{Z},(p, q)=1$ and $2 q+p-2 \geq$ 0 (such level is called admissible). Let $j \in \mathbb{Z}_{+}$.

Then $\hat{\mathfrak{g}}$-module $\overline{V\left(j \omega_{1}\right)} \otimes L_{k}$ is irreducible if $j>2 q+p-2$ and reducible if $j \leq 2 q+p-2$ (here $\omega_{1}$ denotes the fundamental weight for $s l_{2}$ ).

Theorem 2 gives the existence of an infinite family of irreducible modules having infinite-dimensional weight spaces on every admissible rational level.

\section{Preliminaries}

\subsection{Vertex operator algebras and modules.}

Definition 1.1. A vertex operator algebra (VOA) is a $\mathbb{Z}_{+}$-graded vector space $V=\bigoplus_{n \in \mathbb{Z}} V_{n}$ with a sequence of linear operators $\{a(n) \mid n \in \mathbb{Z}\} \subset$ End $V$ associated to every $a \in V$, such that for fixed $a, b \in V, a(n) b=0$ for $n$ sufficiently large. We call the generating series $Y(a, z)=\sum_{n \in \mathbb{Z}} a(n) z^{-n-1} \in$ (End $V$ ) $\left[\left[z, z^{-1}\right]\right]$, vertex operators associated to $a$, satisfy the following axioms:

(1) $Y(a, z)=0$ iff $a=0$.

(2) There is a vacuum vector, which we denote by $\mathbf{1}$, such that

$$
Y(\mathbf{1}, z)=I_{V}\left(I_{V} \text { is the identity of End } V\right) \text {. }
$$

(3) There is a special element $\omega \in V$ (called the Virasoro element), whose vertex operator we write in the form

$$
Y(\omega, z)=\sum_{n \in \mathbb{Z}} \omega(n) z^{-n-1}=\sum_{n \in \mathbb{Z}} L_{n} z^{-n-2},
$$

such that

$$
L_{0} a=n a=(\operatorname{deg} a) a \quad \text { for } a \in V_{n} \text {; }
$$




$$
\begin{gathered}
Y\left(L_{-1} a, z\right)=\frac{d}{d z} Y(a, z) \text { for every } a \in V, \\
{\left[L_{m}, L_{n}\right]=(m-n) L_{m+n}+\delta_{m+n, 0} \frac{m^{3}-m}{12} c,}
\end{gathered}
$$

where $c$ is some constant in $\mathbb{C}$, which is called the rank of $V$.

(4) The Jacobi identity holds, i.e.

$$
\begin{gathered}
z_{0}^{-1} \delta\left(\frac{z_{1}-z_{2}}{z_{0}}\right) Y\left(a, z_{1}\right) Y\left(b, z_{2}\right)-z_{0}^{-1} \delta\left(\frac{-z_{2}+z_{1}}{z_{0}}\right) Y\left(b, z_{2}\right) Y\left(a, z_{1}\right) \\
=z_{2}^{-1} \delta\left(\frac{z_{1}-z_{0}}{z_{2}}\right) Y\left(Y\left(a, z_{0}\right) b, z_{2}\right)
\end{gathered}
$$

for any $a, b \in V$.

The subspace $I$ of $V$ is called ideal if $Y(a, z) b \in I\left[\left[z, z^{-1}\right]\right]$ for every $a \in V, b \in$ $I$. Given an ideal $I$ in $V$ such that $\mathbf{1} \notin I, \omega \notin I$, the quotient $V / I$ admits a natural VOA structure (see [FZ]).

Definition 1.2. Given an $V O A V$, a representation of $V$ (or $V$-module) is a $\mathbb{Z}_{+}$-graded vector space $M=\bigoplus_{n \in \mathbb{Z}_{+}} M(n)$ and a linear map

$$
\begin{gathered}
V \longrightarrow(\text { End } M)\left[\left[z, z^{-1}\right]\right], \\
a \longmapsto Y_{M}(a, z)=\sum_{n \in \mathbb{Z}} a(n) z^{-n-1},
\end{gathered}
$$

satisfying

(1) $a(n) M(m) \subset M(m+\operatorname{deg} a-n-1)$ for every homogeneous element a.

(2) $Y_{M}(1, z)=I_{M}$, and setting $Y_{M}(\omega, z)=\sum_{n \in \mathbb{Z}} L_{n} z^{-n-2}$, we have

$$
\begin{gathered}
{\left[L_{m}, L_{n}\right]=(m-n) L_{m+n}+\delta_{m+n, 0} \frac{m^{3}-m}{12} c} \\
Y_{M}\left(L_{-1} a, z\right)=\frac{d}{d z} Y_{M}(a, z)
\end{gathered}
$$

for every $a \in V$.

(3) The Jacobi identity holds, i.e.

$$
\begin{gathered}
z_{0}^{-1} \delta\left(\frac{z_{1}-z_{2}}{z_{0}}\right) Y_{M}\left(a, z_{1}\right) Y_{M}\left(b, z_{2}\right)-z_{0}^{-1} \delta\left(\frac{-z_{2}+z_{1}}{z_{0}}\right) Y_{M}\left(b, z_{2}\right) Y_{M}\left(a, z_{1}\right) \\
=z_{2}^{-1} \delta\left(\frac{z_{1}-z_{0}}{z_{2}}\right) Y_{M}\left(Y\left(a, z_{0}\right) b, z_{2}\right)
\end{gathered}
$$

for any $a, b \in V$. 
The submodules, quotient modules, irreducible modules, completely reducible modules are defined in the usual way ([FHL]).

Let $M_{i}(i=1,2,3)$ be $V$-modules. Then an intertwining operator of type $\left(\begin{array}{c}M_{3} \\ M_{1}, M_{2}\end{array}\right)$ is defined [FHL] to be a linear map $I(\cdot, z)$ from $M_{1}$ to $\operatorname{Hom}_{\mathbb{C}}\left(M_{2}, M_{3}\right)\{z\}$ such that $I\left(L_{-1} u, z\right)=\frac{d}{d z} I(u, z)$ for $u \in M_{1}$ and a suitably adjusted Jacobi identity holds. Denote by $I\left(\begin{array}{c}M_{3} \\ M_{1}, M_{2}\end{array}\right)$ the space of all intertwining operators of the indicated type. The dimension of this vector space is called the fusion rule of this type. It is well-known that the spaces $I\left(\begin{array}{c}M_{3} \\ M_{1}, M_{2}\end{array}\right)$ and $I\left(\begin{array}{c}M_{3} \\ M_{2}, M_{1}\end{array}\right)$ are isomorphic.

1.2 Zhu's algebra $A(V)$. Let $V$ be a VOA. For any homogeneous element $a \in V$ and for any $b \in V$, following [Z] we define

$$
a * b=\operatorname{Res}_{z} \frac{(1+z)^{\operatorname{deg} a}}{z} Y(a, z) b
$$

Then extend this product bilinearly to the whole space $V$. Let $O(V)$ be the subspace of $V$ linearly spanned by the elements of type

$$
\operatorname{Res}_{z} \frac{(1+z)^{\operatorname{deg} a}}{z^{2}} Y(a, z) b \text { for homogeneous elements } a, b \in V \text {. }
$$

Set $A(V)=V / O(V)$. The multiplication $*$ induces the multiplication on the $A(V)$ and $A(V)$ becomes an associative algebra. The image of $\mathbf{1}$ in $A(V)$ becomes the identity element till the image of $\omega$ is in center of $A(V)$ (see [Z]). Let $M=$ $\oplus_{n \in \mathbb{Z}_{+}} M(n)$ be a $V$-module. For a homogeneous element $a \in V$ we define $o(a)=a(\operatorname{deg} a-1)$. From the definition of $M$ follows that operator $o(a)$ preserves the graduation of $M$.

\section{Theorem 1.1.}

(1) On $\operatorname{End}(M(0))$ we have

$$
o(a) o(b)=o(a * b), \quad o(x)=0
$$

for every $a, b \in V, x \in O(V)$. The top level $M(0)$ is an $A(V)$-module.

(2) Let $U$ be an $A(V)$-module. There exists $V$-module $M$ such that $A(V)$ module $M(0)$ and $U$ are isomorphic.

Thus, we have one-to-one correspondence between irreducible $V$-modules and irreducible $A(V)$-modules.

We have the following consequence from the definition of $A(V)$.

Proposition 1.1. Let $I$ be an ideal of $V$ Assume $\mathbf{1} \notin I, \omega \notin I$. Then the associative algebra $A(V / I)$ is isomorphic to $A(V) /[I]$, where $[I]$ is the image of $I$ in $A(V)$. 


\subsection{Vertex operator algebras associated to affine Lie algebras.}

Let $\mathfrak{g}$ be a finite-dimensional simple Lie algebra over $\mathbb{C}$ and let $(\cdot, \cdot)$ be a nondegenerate symmetric bilinear form on $\mathfrak{g}$. Let $\mathfrak{g}=\mathfrak{n}_{-}+\mathfrak{h}+\mathfrak{n}_{+}$be a triangular decomposition for $\mathfrak{g}$. The affine Lie algebra $\hat{\mathfrak{g}}$ associated with $\mathfrak{g}$ is defined as $\mathfrak{g} \otimes \mathbb{C}\left[t, t^{-1}\right] \oplus \mathbb{C} c \oplus \mathbb{C} d$, where $c$ is the canonical central element $[\mathrm{K}]$ and the Lie algebra structure is given by

$$
\begin{gathered}
{\left[x \otimes t^{n}, y \otimes t^{m}\right]=[x, y] \otimes t^{n+m}+n(x, y) \delta_{n+m, 0} c,} \\
{\left[d, x \otimes t^{n}\right]=n x \otimes t^{n}}
\end{gathered}
$$

for $x, y \in \mathfrak{g}$. We will write $x(n)$ for $x \otimes t^{n}$. We define the subalgebras $\hat{\mathfrak{g}}_{+}$and $\hat{\mathfrak{g}}_{-}$with $\hat{\mathfrak{g}}_{ \pm}=\mathfrak{g} \otimes t^{ \pm 1} \mathbb{C}\left[t^{ \pm 1}\right]$. The Cartan subalgebra $\hat{\mathfrak{h}}$ and subalgebras $\hat{\mathfrak{n}}_{+}, \hat{\mathfrak{n}}_{-}$ of $\hat{\mathfrak{g}}$ are defined by

$$
\hat{\mathfrak{h}}=\mathfrak{h} \oplus \mathbb{C} c \oplus \mathbb{C} d, \quad \hat{\mathfrak{n}}_{ \pm}=\mathfrak{n}_{ \pm} \oplus \mathfrak{g} \otimes t^{ \pm 1} \mathbb{C}\left[t^{ \pm 1}\right] .
$$

Let $\hat{\mathfrak{g}}^{\prime}$ by the subalgebra $\mathfrak{g} \otimes \mathbb{C}\left[t, t^{-1}\right] \oplus \mathbb{C} c$.

Set $\mathfrak{n}_{-}(1)=\mathfrak{n}_{-} \otimes t^{1}$. Let $P_{+}$be the set of all dominant integral weights for g. Let $\Delta$ be a root system for $\hat{\mathfrak{g}}$. Set $\Gamma=\sum_{\varphi \in \Delta} \mathbb{Z} \varphi$.

For a fixed weight $\lambda \in \hat{\mathfrak{h}}^{*}$ let $L(\lambda)$ be the irreducible $\hat{\mathfrak{g}}$-module with the highest weight $\lambda$. Let $\bar{\lambda}$ be a restriction on $\mathfrak{h}$. For a weight $\mu \in P_{+}$, let $V(\mu)$ be the irreducible finite-dimensional highest weight $\mathfrak{g}$-module with the highest weight $\mu$. Let $\overline{V(\mu)}=V(\mu) \otimes \mathbb{C}\left[t, t^{-1}\right]$ be the corresponding loop $\hat{\mathfrak{g}}-$ module of level 0.

Let $P=\mathbb{C}[t] \otimes \mathfrak{g} \oplus \mathbb{C} c$ be upper parabolic subalgebra. Let $U$ be any $\mathfrak{g}$-module. Considering $U$ as a $P$-module, we have the induced module (so called generalized Verma module) $M(k, U)=U(\hat{\mathfrak{g}}) \otimes_{U(P)} U$, where the central element $c$ acts as multiplication with $k \in \mathbb{C}$.

For $\mu \in \mathfrak{h}^{*}$ with $M(\mu)$ we denote Verma module and with $V(\mu)$ its irreducible quotient.

Set $M_{k, \mu}=M(k, V(\mu))$. Let $L_{k, \mu}$ denotes its irreducible quotient. Set $M_{k}=$ $M_{k, 0}, L_{k}=L_{k, 0}$.

Theorem 1.2. ([FZ]) For every $k \neq-h^{\vee}$ (where $h^{\vee}$ denotes dual Coxeter number) $\hat{\mathfrak{g}}$-modules $M_{k}$ and $L_{k}$ have the structure of VOA. Let $U$ be any $\mathfrak{g}-$ module. Then every $M(k, U)$ is a module for $M_{k}$. In particular, $M_{k, \mu}$ is $M_{k}^{-}$ module.

We define the $\mathbb{Z}$-grading on $U(\hat{\mathfrak{g}})$ with

$$
\operatorname{deg} a_{1}\left(-i_{1}\right) \cdots a_{r}\left(-i_{r}\right)=i_{1}+\cdots i_{r}
$$

for every $a_{1}, \ldots a_{r} \in \mathfrak{g}$. Let $y \in U\left(\mathfrak{g}_{-}\right)$such that $\operatorname{deg} y=n$. Let $\mathbf{1}_{k}$ be the vacuum vector in $M_{k}$. Clearly we have that $y \mathbf{1}_{k} \in M_{k}$ and $L_{0}\left(y \mathbf{1}_{k}\right)=(\operatorname{deg} y)\left(y \mathbf{1}_{k}\right)$.

Next we define the projection map $F: U\left(\hat{\mathfrak{g}}_{-}\right) \rightarrow U(\mathfrak{g})$ with

$$
F\left(a_{1}\left(-i_{1}-1\right) \cdots a_{n}\left(-i_{n}-1\right)\right)=(-1)^{i_{1}+\cdots i_{n}} a_{n} a_{n-1} \cdots a_{1},
$$

for every $a_{1}, \ldots, a_{n} \in \mathfrak{g}, i_{1}, \ldots, i_{n} \in \mathbb{Z}_{+}, n \in \mathbb{N}$. 
Theorem 1.3. ([FZ]) The Zhu's algebra $A\left(M_{k}\right)$ is canonically isomorphic to $U(\mathfrak{g})$ and the isomorphism $\bar{F}: A\left(M_{k}\right) \rightarrow U(\mathfrak{g})$ is given by:

$$
\bar{F}\left(a_{1}\left(-i_{1}-1\right) \cdots a_{n}\left(-i_{n}-1\right) \mathbf{1}_{k}+O\left(M_{k}\right)\right)=F\left(a_{1}\left(-i_{1}-1\right) \cdots a_{n}\left(-i_{n}-1\right)\right)
$$

for every $a_{1}, \cdots, a_{n} \in \mathfrak{g}$ and every $i_{1}, \cdots, i_{n} \in \mathbb{Z}_{+}$.

We know that for every $\lambda \in \mathfrak{h}^{*}$ and $k \neq-h^{\vee} L_{k, \lambda}$ is a $M_{k}$-module. One can show that $L_{k, \lambda}=\coprod_{n \in \mathbb{Z}_{+}} L_{k, \lambda}(n)$ and $L_{0} \mid L_{k, \lambda}(n)=\left(n+h_{k, \lambda}\right) \operatorname{Id}$, where $h_{k, \lambda}=\frac{(\lambda, \lambda+2 \rho)}{2\left(k+h^{\vee}\right)}($ see $[\mathrm{DL}])$. Then $\hat{\mathfrak{g}}^{\prime}$-module $L_{k, \lambda}$ becomes $\hat{\mathfrak{g}}=\hat{\mathfrak{g}}^{\prime}+\mathbb{C} d$-module with $d=L_{0}-h_{\ell, \lambda}$.

Let $L_{k, \mu}, L_{k, \lambda}, L_{k, \nu}$ are $M_{k}$-modules. Set $h_{1}=h_{k, \mu}, h_{2}=h_{k, \lambda} h_{3}=h_{k, \nu}$. Let $I(\cdot, z)$ be an intertwining operator from the space $I\left(\begin{array}{c}L_{k, \nu} \\ L_{k, \mu}, L_{k, \lambda}\end{array}\right)$. We define

$$
I^{\circ}\left(u^{1}, z\right)=z^{h_{1}+h_{2}-h_{3}} I\left(u^{1}, z\right)=\sum_{n \in \mathbb{Z}} u^{1}(n) z^{-n-1}
$$

for $u^{1} \in L_{k, \mu}$. Then $I^{\circ}(\cdot, z)$ can be considered as a linear map from $\mathbb{C}\left[t, t^{-1}\right] \otimes$ $L_{k, \mu} \otimes L_{k, \lambda}$ to $L_{k, \nu}$. Let $\phi_{I}$ denotes a restriction of $I^{\circ}(\cdot, z)$ on $\mathbb{C}\left[t, t^{-1}\right] \otimes V(\mu) \otimes$ $L_{k, \lambda}$. By using Jacobi identity one can easily obtain the following relations

$$
\begin{aligned}
{[a(m), I(u, z)] } & =z^{m} I(a u, z) ; \\
{\left[L_{m}, I(u, z)\right] } & =\left((m+1) h_{1}+z \frac{d}{d z}\right) I(u, z)
\end{aligned}
$$

for $a \in \mathfrak{g} \subset M_{k}, u \in V(\mu), m \in \mathbb{Z}$.

This implies that $\phi_{I} \in \operatorname{Hom}_{\hat{\mathfrak{g}}}\left(\overline{V(\mu)} \otimes L_{k, \lambda}, L_{k, \nu}\right)$. We have the following lemma:

Lemma 1.1. Linear map $\phi$ defined with

$$
\begin{gathered}
\phi: \quad I\left(\begin{array}{c}
L_{k, \nu} \\
L_{k, \mu}, L_{k, \lambda}
\end{array}\right) \rightarrow \operatorname{Hom}_{\hat{\mathfrak{g}}}\left(\overline{V(\mu)} \otimes L_{k, \lambda}, L_{k, \nu}\right) ; \\
I(\cdot, z) \mapsto \phi_{I}
\end{gathered}
$$

is injective.

Proof. Let $\phi_{I}=0$. Then $I^{\circ} \equiv 0$ on $V(\mu)$. Since $V(\mu)$ generate $L_{k, \mu}$, from Jacobi identity follows $I^{\circ}=0$, and we get $I=0$.

Remark 1.1. H.-Li in [Li] proved that if $k \in \mathbb{N}$ and $L_{k, \lambda}, L_{k, \mu}, L_{k, \nu}$ are $L_{k^{-}}$ modules, then the map $\phi$ is an isomorphism.

Lemma 1.2. Assume that the space $I\left(\begin{array}{c}L_{k, \nu} \\ L_{k, \mu}, L_{k, \lambda}\end{array}\right)$ is nontrivial. Then the $\hat{\mathfrak{g}}-$ module $\overline{V(\mu)} \otimes L_{k, \lambda}$ is reducible.

Proof. Let $I \in I\left(\begin{array}{c}L_{k, \nu} \\ L_{k, \mu}, L_{k, \lambda}\end{array}\right)$ and $I \neq 0$. Then $\phi_{I}: \overline{V(\mu)} \otimes L_{k, \lambda} \rightarrow L_{k, \nu}$ is a nontrivial $\hat{\mathfrak{g}}$-homomorphism. Assume that $\overline{V(\mu)} \otimes L_{k, \lambda}$ is irreducible. Then $\phi_{I}$ is injective $\hat{\mathfrak{g}}$-homomorphism, which implies that $\overline{V(\mu)} \otimes L_{k, \lambda}$ is isomorphic to $L_{k, \nu}$. This is a contradiction, since the weight spaces of $\overline{V(\mu)} \otimes L_{k, \lambda}$ are infinite-dimensional and the weight spaces of $L_{k, \nu}$ are finite-dimensional. 
Proposition 1.2. Let $\mu \in P_{+}$such that $V(\mu)$ is a module for Zhu's algebra $A\left(L_{k}\right)$. Then the $\hat{\mathfrak{g}}-$ module $\overline{V(\mu)} \otimes L_{k}$ is reducible.

Proof. Since $V(\mu)$ is $A\left(L_{k}\right)$, then $L_{k, \mu}$ is $L_{k}$-module. This implies that the space

$$
I\left(\begin{array}{c}
L_{k, \mu} \\
L_{k, \mu}, L_{k}
\end{array}\right) \cong I\left(\begin{array}{c}
L_{k, \mu} \\
L_{k}, L_{k, \mu}
\end{array}\right)
$$

is nontrivial. Then the proposition follows from Lemma 1.2.

Remark 1.2. In what follows we will prove that if $V(\mu)$ is not a $A\left(L_{k}\right)$-module, then the $\hat{\mathfrak{g}}-$ module $\overline{V(\mu)} \otimes L_{k}$ is irreducible.

\section{Tensor products $\overline{V(\mu)} \otimes L_{k}$}

In this section let $\mu \in P_{+}$and $\lambda \in \hat{\mathfrak{h}}^{*}$. We will consider the tensor product $\overline{V(\mu)} \otimes L(\lambda)$. Let $v_{\lambda}$ be a highest weight vector in $L(\lambda)$ and let $\Omega_{\mu}$ a highest weight vector in $V(\mu)$. For $n \in \mathbb{Z}$ and $Z \in V(\mu)$ set $Z_{n}=Z \otimes t^{n}$. Let $\Omega_{\mu, n}=\left(\Omega_{\mu}\right)_{n}$ and $\Omega_{w \mu, n}=\left(\Omega_{w \mu}\right)_{n}$ ( $w$ here denotes the element of maximal length in the Weyl group of $\mathfrak{g})$. Let $V_{n}$ be the $\hat{\mathfrak{g}}$-submodule generated by the element $\Omega_{\mu, n} \otimes v_{\lambda}$.

We shall need the following lemma from $[\mathrm{CP}]$ :

Lemma 2.1. (Lemma 2.1 from $[\mathrm{CP}]$ ) As an $\hat{\mathfrak{g}}-$ module $\overline{V(\mu)} \otimes L(\lambda)$ is generated by each of the sets

$$
\left\{\Omega_{\mu, n} \otimes v_{\lambda}, \quad n \in \mathbb{Z}\right\}, \quad\left\{\Omega_{w \mu, n} \otimes v_{\lambda}, \quad n \in \mathbb{Z}\right\} .
$$

\section{Corollary 2.1.}

(1) $V_{n} \supseteq V_{n+1}$ for all $n \in \mathbb{Z}$,

(2) $\cup_{n \in \mathbb{Z}} V_{n}=\overline{V(\mu)} \otimes L(\lambda)$.

Proof. For (1) it is enough to see that there is $h \in \mathfrak{h}$ such that

$$
h(1)\left(\Omega_{\mu, n} \otimes v_{\lambda}\right)=\Omega_{\mu, n+1} \otimes v_{\lambda} .
$$

Statment (2) follows from Lemma 2.1.

The following lemma was proved in $[\mathrm{A}]$.

Lemma 2.2. (Lemma 1.1 from $[\mathrm{A}]$ ) For $x_{1}, \ldots, x_{r} \in \mathfrak{g} ; j_{1}, \ldots, j_{r} \in \mathbb{Z}, r \in \mathbb{N}$ we have

$$
\begin{gathered}
x_{1}\left(j_{1}\right) \cdots x_{r}\left(j_{r}\right) .\left(\Omega_{\mu, n} \otimes v_{\lambda}\right) \in \\
\sum_{\substack{k=0 \\
k_{1}, \ldots, k_{r} \in\{0,1\} \\
k_{1}+\cdots+k_{r}=k}}(-1)^{k} \Omega_{\mu, n+k} \otimes x_{1}\left(j_{1}-k_{1}\right) \ldots x_{r}\left(j_{r}-k_{r}\right) v_{\lambda} \\
+V_{n+1}, \quad n \in \mathbb{Z} .
\end{gathered}
$$

For $v \in \overline{V(\mu)} \otimes L(\lambda)$ set $[v]=v+V_{n+1}$. Clearly, $U(\hat{\mathfrak{g}})$-module $V_{n} / V_{n+1}$ is generated by $\left[\Omega_{\mu, n} \otimes v_{\lambda}\right]$. 
Proposition 2.1. (Proposition 1.1 from $[\mathrm{A}]$ ) For every $n \in \mathbb{Z}$ the $\hat{\mathfrak{g}}$-module $V_{n} / V_{n+1}$ is in the category $\mathcal{O}$.

Let $J_{k}$ be the maximal $\hat{\mathfrak{g}}$-submodule in $M_{k}$. Then $L_{k}=M_{k} / J_{k}$ is irreducible highest weight $\hat{\mathfrak{g}}-$ module with the highest weight $k \Lambda_{0}$. Let $v_{k}=\mathbf{1}_{k}+J_{k}$ be the highest weight vector in $L_{k}$. We are interested in tensor products $\overline{V(\mu)} \otimes L_{k}$. We have the following:

Proposition 2.2. Let $\mu \in P_{+}$and $k \in \mathbb{C}$. Assume that $V_{n} / V_{n+1} \neq 0$. Then we have the following:

(1) $V_{n} / V_{n+1}$ is the highest weight $\hat{\mathfrak{g}}$-module and $\left[\Omega_{\mu, n} \otimes v_{k}\right]$ is the highest weight vector.

(2) $\left[Z_{n} \otimes v_{k}\right] \neq 0$ for every $Z \in \mathfrak{g}, Z \neq 0$.

Proof. (1) Since $V_{n} / V_{n+1}$ is in the category $\mathcal{O}$ and $\left[\Omega_{\mu, n} \otimes v_{k}\right]$ generates $V_{n} / V_{n+1}$, it sufficies to prove that $\left[\Omega_{\mu, n} \otimes v_{k}\right]$ is the highest weight vector.

Since $\hat{\mathfrak{n}}_{+} v_{k}=\mathfrak{n}_{-} v_{k}=0, \mathfrak{n}_{+} \Omega_{\mu, n}=0$, then by using Lemma 2.2 we have that

$$
\begin{gathered}
\left(\hat{\mathfrak{n}}_{+}-\mathfrak{n}_{-}(1)\right)\left[\Omega_{\mu, n} \otimes v_{k}\right]=0, \\
\mathfrak{n}_{-}(1)\left[\Omega_{\mu, n} \otimes v_{k}\right]=-\left[\Omega_{\mu, n+1} \otimes \mathfrak{n}_{-} v_{k}\right]=0 .
\end{gathered}
$$

This implies that $\hat{\mathfrak{n}}_{+}\left[\Omega_{\mu, n} \otimes v_{k}\right]=0$, and we conclude that $\left[\Omega_{\mu, n} \otimes v_{k}\right]$ is the highest weight vector in $V_{n} / V_{n+1}$.

(2) Assume now that there is $Z \in \mathfrak{g}, Z \neq 0$ such that $\left[Z_{n} \otimes v_{k}\right]=0$. Since the $U(\mathfrak{g})$-module $V(\mu)$ is irreducible we can choose $x \in U(\mathfrak{g})$ such that $x Z=\Omega_{\mu}$. Then we have that $\left[\Omega_{\mu, n} \otimes v_{k}\right]=x\left[Z_{n} \otimes v_{k}\right]=0$. This contradicts the fact that $V_{n} / V_{n+1} \neq 0$.

Lemma 2.3. Let $n \in \mathbb{Z}, r \in \mathbb{Z}_{+}$and $Z \in V(\mu)$. For every $n_{1}, n_{2}, \ldots, n_{r} \in \mathbb{Z}_{+}$ and $a_{1}, a_{2}, \ldots, a_{r} \in \mathfrak{g}$ we have

$$
Z_{n+N} \otimes a_{1}\left(-n_{1}-1\right) \cdots a_{r}\left(-n_{r}-1\right) v_{k} \in V_{n}
$$

and

$$
Z_{n+N} \otimes a_{1}\left(-n_{1}-1\right) \cdots a_{r}\left(-n_{r}-1\right) v_{k} \equiv(-1)^{r}\left(a_{r} \cdots a_{1} Z\right)_{n} \otimes v_{k} \bmod V_{n+1},
$$

where $N=n_{1}+n_{2}+\cdots+n_{r}+r$.

Proof. First we notice that $R=U(\mathfrak{g})\left(\Omega_{\mu, m} \otimes v_{k}\right)$ is finite-dimensional $U(\mathfrak{g})$ module isomorphic to $V(\mu)$. This implies that $Z_{m} \otimes v_{k} \in V_{m}$ for every $Z \in V(\mu)$ and $m \in \mathbb{Z}$.

We prove the lemma by induction on $r \in \mathbb{Z}_{+}$. For $r=1$ we have

$$
Z_{n+n_{1}+1} \otimes a_{1}\left(-n_{1}-1\right) v_{k}=a_{1}\left(-n_{1}-1\right)\left(Z_{n+n_{1}+1} \otimes v_{k}\right)-\left(a_{1} Z\right)_{n} \otimes v_{k} .
$$


Since $Z_{n+n_{1}+1} \otimes v_{k} \in V_{n+n_{1}+1} \subset V_{n+1}$, we obtain that

$$
Z_{n+n_{1}+1} \otimes a_{1}\left(-n_{1}-1\right) v_{k} \equiv\left(a_{1} Z\right)_{n} \otimes v_{k} \bmod V_{n+1} .
$$

Assume that the result holds for $r \in \mathbb{Z}_{+}$. We have

$$
\begin{aligned}
& Z_{n+N_{0}} \otimes a_{0}\left(-n_{0}-1\right) a_{1}\left(-n_{1}-1\right) \cdots a_{r}\left(-n_{r}-1\right) v_{k} \\
= & a_{0}\left(-n_{0}-1\right)\left(Z_{n+N_{0}} \otimes a_{1}\left(-n_{1}-1\right) \cdots a_{r}\left(-n_{r}-1\right) v_{k}\right) \\
& -\left(a_{0} Z\right)_{n+N} \otimes a_{1}\left(-n_{1}-1\right) \cdots a_{r}\left(-n_{r}-1\right) v_{k},
\end{aligned}
$$

where $N_{0}=n_{0}+n_{1}+\cdots n_{r}+r+1, N=n_{1}+\cdots n_{r}+r$.

By induction hypothesis follows

$$
Z_{n+N_{0}} \otimes a_{1}\left(-n_{1}-1\right) \cdots a_{r}\left(-n_{r}-1\right) v_{k} \in V_{n+n_{0}+1} \subset V_{n+1},
$$

and we have that

$$
\begin{gathered}
Z_{n+N_{0}} \otimes a_{0}\left(-n_{0}-1\right) a_{1}\left(-n_{1}-1\right) \cdots a_{r}\left(-n_{r}-1\right) v_{k} \\
\equiv-\left(a_{0} Z\right)_{n+N} \otimes a_{1}\left(-n_{1}-1\right) \cdots a_{r}\left(-n_{r}-1\right) v_{k} \bmod V_{n+1} .
\end{gathered}
$$

By the induction hypothesis follows that $\left(a_{0} Z\right)_{n+N} \otimes a_{1}\left(-n_{1}-1\right) \cdots a_{r}\left(-n_{r}-1\right) v_{k} \equiv(-1)^{r+1}\left(a_{r} \cdots a_{1} a_{0} Z\right)_{n} \otimes v_{k} \bmod V_{n+1}$ and the lemma holds.

Lemma 2.4. Let $n \in \mathbb{Z}, d \in \mathbb{Z}_{+}$and $Z \in V(\mu)$. Let $y \in U\left(\hat{\mathfrak{g}}_{-}\right)$such that $\operatorname{deg} y=d$. Then we have

$$
\left[Z_{n+d} \otimes y v_{k}\right]=(-1)^{d}\left[(F(y) Z)_{n} \otimes v_{k}\right]
$$

Proof. Let

$$
y=\sum_{i=1}^{t} a_{j_{1}}^{(i)}\left(-j_{1}^{(i)}-1\right) \cdots a_{j_{l_{i}}}^{(i)}\left(-j_{l_{i}}^{(i)}-1\right) v_{k},
$$

where $a_{j_{1}}^{(i)}, \ldots, a_{j_{l_{i}}}^{(i)} \in \mathfrak{g}, l_{i}, j_{1}^{(i)}, \ldots j_{l_{i}}^{(i)} \in \mathbb{Z}_{+}$such that

$$
j_{1}^{(i)}+\cdots+j_{l_{i}}^{(i)}+l_{i}=d, \quad \forall i=1, \ldots, t .
$$

By using Lemma 2.3 we obtain

$$
\begin{gathered}
{\left[Z_{n+d} \otimes y v_{k}\right]=\sum_{i=1}^{t}(-1)^{l_{i}}\left[\left(a_{j_{l_{i}}}^{(i)} \cdots a_{j_{1}}^{(i)} Z\right)_{n} \otimes v_{k}\right]} \\
=\sum_{i=1}^{t}(-1)^{l_{i}+j_{1}^{(i)}+\ldots j_{l_{i}}^{(i)}}\left[\left(F\left(a_{j_{1}}^{(i)}\left(-j_{1}^{(i)}-1\right) \cdots a_{j_{l_{i}}}^{(i)}\left(-j_{l_{i}}^{(i)}-1\right)\right) Z\right)_{n} \otimes v_{k}\right] \\
=(-1)^{d}\left[(F(y) Z)_{n} \otimes v_{k}\right] .
\end{gathered}
$$

Let $J_{k}$ be the maximal submodule of $M_{k}$. The following lemma follows from definition of the maximal submodule $J_{k}$. 
Lemma 2.5. Let $w \in J_{k}$. Then $w$ can be written in the form $w=\sum_{i=1}^{s} w_{i}$, where $w_{i} \in J_{k}$ and $L_{0} w_{i}=d_{i} w_{i}$ for some $d_{i} \in \mathbb{Z}_{+}, i=1, \ldots, s$.

Proposition 2.3. Assume that $V_{n} / V_{n+1}$ is a nontrivial subquotient of $\hat{\mathfrak{g}}$-module $\overline{V(\mu)} \otimes L_{k}$. Then $V(\mu)$ is a module for Zhu's algebra $A\left(L_{k}\right)$.

Proof. The maximal $\hat{\mathfrak{g}}$-submodule $J_{k}$ of $M_{k}$ is an ideal in vertex operator algebra $M_{k}$. Then $A\left(J_{k}\right)$ is a two-sided ideal in $A\left(M_{k}\right)$. For $w \in M_{k}$ set $\bar{w}=w+$ $O\left(M_{k}\right) \in A\left(M_{k}\right)$. By using isomorphism $\bar{F}: A\left(M_{k}\right) \rightarrow U(\mathfrak{g})$ (Theorem 1.3) and Proposition 1.1 we see that $A\left(J_{k}\right)$ is isomorphic to the two-sided ideal in $U(\mathfrak{g})$ generated by the set $\left\{\bar{F}(\bar{w}), w \in J_{k}\right\}$. Now we will show that $V(\mu)$ is $A\left(L_{k}\right)-$ module. Since $A\left(L_{k}\right)=A\left(M_{k}\right) / A\left(J_{k}\right)$, it suffices to prove that $\bar{F}(\bar{w}) Z=0$ for every $w \in J_{k}$ and $Z \in V(\mu)$.

Let $w \in J_{k}$ and $Z \in V(\mu)$. Then Lemma 2.5 implies that $w=w_{1}+\cdots+w_{s}$, where $w_{i} \in J_{k}$ and $L_{0}\left(w_{i}\right)=d_{i} w_{i}, d_{i} \in \mathbb{Z}_{+}, i=1, \ldots, s$. Let $y_{i} \in U\left(\hat{\mathfrak{g}}_{-}\right)$such that $w_{i}=y_{i} \mathbf{1}_{k}, \operatorname{deg} y_{i}=d_{i}, i=1, \ldots, s$. We have that

$$
\bar{F}(\bar{w}) Z=\sum_{i=1}^{s} F\left(y_{i}\right) Z .
$$

Since $y_{i} v_{k}=0$ in $L_{k}$, by using Lemma 2.4 we have that

$$
\left[\left(F\left(y_{i}\right) Z\right)_{n} \otimes v_{k}\right]=(-1)^{d_{i}}\left[Z_{n+d_{i}} \otimes y_{i} v_{k}\right]=0
$$

for $i=1, \ldots, s$. This implies that

$$
\left[(\bar{F}(\bar{w}) Z)_{n} \otimes v_{k}\right]=0 .
$$

Now Proposition $2.2(2)$ gives $\bar{F}(\bar{w}) Z=0$.

\section{Irreducibility of tensor products $\overline{V(\mu)} \otimes L_{k}$}

We shall need the following fundamental result from $[\mathrm{CP}]$.

Proposition 3.1. (Proposition 2.5 from [CP]) If a module of type $\overline{V(\mu)} \otimes L(\lambda)$ is cyclic on each of the elements $\Omega_{\mu, n} \otimes v_{\lambda}, n \in \mathbb{Z}$, then $\overline{V(\mu)} \otimes L(\lambda)$ is irreducible.

Remark 3.1. The statement of this proposition is true for every $\lambda \in \hat{\mathfrak{h}}^{*}$ (see note after Proposition 2.5 in $[\mathrm{CP}]$ ). In $[\mathrm{CP}]$ this result is applied to the case of integrable modules and in $[\mathrm{A}]$ to the modules at the critical level.

Theorem 3.1. Assume that $\mu \in P_{+}$such that $V(\mu)$ is not $A\left(L_{k}\right)-$ module. Then $\hat{\mathfrak{g}}$-module $\overline{V(\mu)} \otimes L_{k}$ is irreducible.

Proof. In view of Proposition 3.1 it suffices to prove that $V_{n} / V_{n+1}=0$ for all $n \in \mathbb{Z}$. Assume that $V_{n} / V_{n+1}$ is non-zero. Then Proposition 2.3 implies that $V(\mu)$ is $A\left(L_{k}\right)$-module, contradicting the fact that $V(\mu)$ is not $A\left(L_{k}\right)$-module. This proves that $V_{n} / V_{n+1}=0$.

Theorem 3.2. $L_{k, \mu}, \mu \in P_{+}$, is $L_{k}$-module if and only if $\hat{\mathfrak{g}}-\operatorname{module} \overline{V(\mu)} \otimes L_{k}$ is reducible. 


\section{Some general results}

In this section we study the tensor products $\overline{V(\mu)} \otimes L_{k}$ for general simple Lie algebra $\mathfrak{g}$. We list all cases for which it is possible to decide on irreducibility of $\hat{\mathfrak{g}}$-modules $\overline{V(\mu)} \otimes L_{k}$.

First we will consider the case $k \in \mathbb{N}$. Let $e_{\theta}$ be the element corresponding to the highest $\operatorname{root} \theta$.

Theorem 4.1. ([FZ]) Let $k \in \mathbb{N}$. Then $A\left(L_{k}\right)=U(\mathfrak{g}) /\left\langle e_{\theta}^{k+1}\right\rangle$. The irreducible $A\left(L_{k}\right)$-modules are exactly $V(\mu), \mu \in P_{+},(\mu, \theta) \leq k$.

Theorem 4.2. Let $\mu \in P_{+}$and $k \in \mathbb{N}$. Then the $\hat{\mathfrak{g}}$-module $\overline{V(\mu)} \otimes L_{k}$ is reducible if $(\mu, \theta) \leq k$ and irreducible if $(\mu, \theta)>k$.

Remark 4.1. The irreducibility result from Theorem 4.2 is part of the result obtained in $[\mathrm{CP}]$.

We recall the following result for representations of critical level obtained by the author in $[\mathrm{A}]$.

Theorem 4.3. The $\hat{\mathfrak{g}}-$ module $\overline{V(\mu)} \otimes L_{-h^{\vee}}$ is irreducible for every $\mu \in P_{+}$.

Now we recall the following well known result obtained in [KL] for the algebras of the type $A, D, E$.

Lemma 4.1. $[\mathrm{KL}]$ Let $k \notin \mathbb{Q}$ or $k<-h^{\vee}$. Then the generalized Verma module $M_{k}$ is irreducible, i.e. $L_{k}=M_{k}$.

Theorem 4.4. Let $k \notin \mathbb{Q}$ or $k<-h^{\vee}$. Then the $\hat{\mathfrak{g}}$-module $\overline{V(\mu)} \otimes L_{k}$ is reducible for every $\mu \in P_{+}$.

Proof. From Lemma 4.1 follows that $L_{k}=M_{k}$, and we have that $A\left(L_{k}\right)=$ $A\left(M_{k}\right)=U(\mathfrak{g})$. This implies that for every $\mu \in P_{+} V(\mu)$ is a $A\left(L_{k}\right)$-module. Now Proposition 1.2 gives that the $\hat{\mathfrak{g}}-$ module $\overline{V(\mu)} \otimes L_{k}$ is reducible.

Remark 4.2. We see that we can solve the question of irreducibility for modules $\overline{V(\mu)} \otimes L_{k}$ in the following cases : $k \notin \mathbb{Q}, k<-h^{\vee}, k=-h^{\vee}, k \in \mathbb{N}$. In what follows we study these modules for $k+h^{\vee} \in \mathbb{Q}_{>0}$, and we will solve the irreducibility question for affine Lie algebra $A_{1}^{(1)}$.

\section{The case $\mathfrak{g}=s l_{2}$}

Let now $\mathfrak{g}=s l(2, \mathbb{C})$ with generators $e, f, h$ and relations: $[h, f]=-2 f$, $[h, e]=2 e,[e, f]=h$. Let $\Lambda_{0}, \Lambda_{1}$ denote the fundamental weights for $\hat{\mathfrak{g}}$, and $\omega_{1}$ the fundamental weight for $\mathfrak{g}$.

Admissible representations are irreducible highest weight representations whose highest weight is an admissible weight $([\mathrm{KW} 1])$. Admissible weights are defined as follows. 
Definition 5.1. We call a weight $\lambda$ admissible if it satisfies the following two conditions.

(1) $\left\langle\lambda+\hat{\rho}, \alpha^{\vee}\right\rangle \notin\{0,-1,-2,-3, \cdots\} \quad$ for all real positive coroots $\alpha^{\vee}$,

(2) $\mathbb{Q} R^{\lambda}=\mathbb{Q} \Pi^{\vee}$

where $\hat{\rho}$ is the sum of all fundamental weights, $\Pi^{\vee}$ is the set of simple coroots and

$$
R^{\lambda}=\left\{\alpha^{\vee}: \text { a positive real coroot } \mid\left\langle\lambda+\rho, \alpha^{\vee}\right\rangle \in \mathbb{Z}\right\}
$$

The classification of admissible weights is given in $[\mathrm{KW} 2]$. We recall this classification in the case of affine Lie algebra $A_{1}^{(1)}$.

Definition 5.2. A rational number $k=p / q \in \mathbb{Q}$ is called admissible if $q \in \mathbb{N}$, $p \in \mathbb{Z},(p, q)=1$ and $2 q+p-2 \geq 0$.

Proposition 5.1. Let $k=p / q \in \mathbb{Q}$ be admissible. Set $t=k+2$. The set of all admissible weights at the level $k$ is

$P^{k}=\left\{(k-n+m t) \Lambda_{0}+(n-m t) \Lambda_{1}, m, n \in \mathbb{Z}_{+}, n \leq 2 q+p-2, m \leq q-1\right\}$.

When $k$ is admissible then $\hat{\mathfrak{g}}$-module $L_{k}=L\left(k \Lambda_{0}\right)$ carries the structure of VOA. Moreover, there is a vector $Q \in U(\mathfrak{g}), Q \neq 0$, such that the Zhu's algebra $A\left(L_{k}\right)$ is isomorphic to $U(\mathfrak{g}) /\langle Q\rangle$, where $\langle Q\rangle$ is a two-sided ideal in $U(\mathfrak{g})$ generated by $Q$.

We recall the following results from $[\mathrm{AM}]$.

Lemma 5.1. (Proposition 3.5.2 from $[\mathrm{AM}]$ ) The set $\left\{V(\bar{\lambda}) \mid \lambda \in P^{k}\right\}$ provides all irreducible $A\left(L_{k}\right)$-modules from the category $\mathcal{O}$.

Lemma 5.2. (Theorem 3.5.3 from $[\mathrm{AM}]$ ) The set $\left\{L(\lambda) \mid \lambda \in P^{k}\right\}$ provides all irreducible $L_{k}$-modules from the category $\mathcal{O}$.

From the Lemma 5.1 we get:

Theorem 5.1. All irreducible finite-dimensional $A\left(L_{k}\right)$-modules are exactly

$$
V\left(j \omega_{1}\right), \quad j \in\{0,1, \ldots, 2 q+p-2\}
$$

Now Theorem 5.1 and Theorem 3.1 imply the following.

Theorem 5.2. Let $j \in \mathbb{Z}_{+}$, and let $k=p / q$ be admissible. Then $\hat{\mathfrak{g}}-$ module $\overline{V\left(j \omega_{1}\right)} \otimes L_{k}$ is irreducible if $j>2 q+p-2$ and reducible if $j \leq 2 q+p-2$.

The irreducibility is not affected if we twist the representation by the nontrivial diagram automorphism of $A_{1}^{(1)}$. This automorphism is determined by $e(n) \mapsto f(n+1), f(n) \mapsto e(n-1)$ and $h(n) \mapsto-h(n)+\delta_{n, 0} c$, and we obtain the following. 
Corollary 5.1. Let $j \in \mathbb{Z}_{+}$, and let $k=p / q$ be admissible. Then $\hat{\mathfrak{g}}-$ module $\overline{V\left(j \omega_{1}\right)} \otimes L\left(k \Lambda_{1}\right)$ is irreducible if $j>2 q+p-2$ and reducible if $j \leq 2 q+p-2$.

Remark 5.1. For every $M$ weight $\hat{\mathfrak{g}}-$ module let $P(M)$ be the set of weights of $M$. An irreducible weight module $M$ is called dense if $P(M)=\lambda+\Gamma$ for some $\lambda \in \hat{\mathfrak{h}}^{*}$, and non-dense otherwise (see $[\mathrm{F}]$ ). Irreducible modules which are realized as tensor product of irreducible highest weight modules with loop modules are dense. Theorem 5.2 shows that at every admissible rational level exist dense $A_{1}^{(1)}$-modules with all infinite-dimensional weight spaces.

We have the following conjecture.

Conjecture 5.1. Let $\mathfrak{g}$ be any simple Lie algebra. Assume that $k \Lambda_{0}$ is admissible weight and $\mu \in P_{+}$. Define $\hat{\mu}=k \Lambda_{0}+\mu$. Then the $\hat{\mathfrak{g}}-$ module $\overline{V(\mu)} \otimes L_{k}$ is irreducible if and only if $\hat{\mu}$ is not an admissible weight.

\section{References}

[A] D. Adamović, New irreducible modules for affine Lie algebras at the critical level, Internat. Math. Res. Notices 6 (1996), 253-261.

[AM] D. Adamović and A. Milas, Vertex operator algebras associated to the modular invariant representations for $A_{1}^{(1)}$, Math. Res. Lett. 2 (1995), 563-575.

[CP] V. Chari and A. Pressley, A new family of irreducible, integrable modules for affine Lie algebras, Math. Ann. 277 (1987), 543-562.

[DL] C. Y. Dong and J. Lepowsky, Generalized vertex algebras and relative vertex operators, Birkhäuser, Boston, 1993.

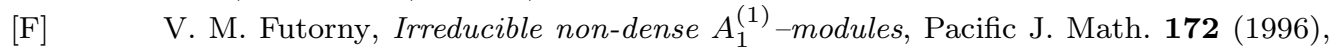
83-99.

[FZ] I. B. Frenkel and Y. Zhu, Vertex operator algebras associated to representations of affine and Virasoro algebras, Duke Math. J. 66 (1992), 123-168.

[FHL] I. B. Frenkel, Y.-Z. Huang, and J. Lepowsky, An axiomatic approach to Vertex operator algebras and modules, Mem. Amer. Math. Soc. 104 (1993).

[K] V. G. Kac, Infinite dimensional Lie algebras, Birkhäuser, Basel, 1983.

[KL] D. Kazhdan and G. Lusztig, Tensor structures arising from affine Lie algebras I, J. Amer. Math. Soc. 64 (1993), 905-947.

[KW 1] V. G. Kac and M. Wakimoto, Modular invariant representations of infinite dimensional Lie algebras and superalgebras, Proc. Natl. Acad. Sci. USA 85 (1988), 4956-4960.

[KW 2] _ Classification of modular invariant representations of affine algebras, Infinite-dimensional Lie algebras and groups (Luminy-Marseille, 1988), 138-177, Adv. Ser. Math. Phys., vol. 7, World Sci. Publishing, Teaneck, NJ, 1989.

[Li] H.-Li, Representation theory and tensor product theory of vertex operator algebras (1994), PH. D. dissertation, Rutgers University.

$[\mathrm{MP}] \quad$ A. Meurman and M. Primc, Annihilating fields for $s_{2}(\mathbb{C})^{\tilde{a}}$ and combinatorial identities, Trans. Amer. Math. Soc. (to appear).

[Z] Y. Zhu, Vertex operator algebras, elliptic function and modular forms (1990), Ph. D. dissertation, Yale University.

Dept. of Math., University of Zagreb, Bijenička 30, 10000 Zagreb, CROATiA

E-mail address: adamovic@cromath.math.hr 